\title{
Ab-initio positioning of the valence and conduction bands of bulk photocatalysts: Proposition of absolute reference energy
}

\author{
Tilak Das $^{\dagger *}$, Xavier Rocquefelte ${ }^{\ddagger *}$, and Stéphane Jobic \\ Université de Nantes, CNRS, Institut des Matériaux Jean Rouxel, IMN, F-44000, Nantes, France
}

KEYWORDS: Photocatalysts, Band Alignment, HOMO and LUMO, Ab-initio, Hybrid Density Functional Theory, Absolute Vacuum Energy, Mean Absolute Error, Standard Deviation.

\begin{abstract}
Finding absolute reference energy from first-principles calculations to align redox positions of valence band top and hence conduction band bottom of bulk inorganic photocatalysts is still a challenge. A theoretical methodology is proposed herein based on first-principles calculations using state-of-art hybrid density functional theory from Heyd-ScuseriaErnzerhof. Both oxides and non-oxides materials, known for their potential capability for photocatalysis i.e. rutile- and anatase $\mathrm{TiO}_{2}$, wurtzite $\mathrm{ZnO}$, rutile $\mathrm{SnO}_{2}$, blende phase of GaP, GaAs, InP, $\mathrm{ZnTe}$, CdS, CdSe, and SiC, have been studied. The calculated band-edges around the fundamental band-gap of these compounds are realigned, reference to the corrected vacuum energy level from the probe's core energy state i.e. the $1 \mathrm{~s}^{2}$ state of an unreactive helium atom. Calculated $a b$-initio positioning of valence and conduction band extrema are compared to the available experimental data, and our prediction is best fitted within mean absolute error of $0.2 \mathrm{eV}$.
\end{abstract}

\section{INTRODUCTION}

Defining a proper theoretical strategy to align the redox levels of semiconductors and insulators on an absolute reference energy scale is of great importance in physics, chemistry and electrochemistry. ${ }^{1}$ Namely, many technological applications, such as photodiodes, LEDs, MOSFETs or MESFETs, are based on the fine tuning of the energy levels at the junction between two semiconductors. More specifically, it is crucial to characterize and control their band energy-offsets, which arise from the discontinuity of their valence and conduction bandedges at their interface. ${ }^{2-3}$ Similarly, many renewable energy applications are based on hetero-junctions of semiconductors. In the current scenario of downing of fossil fuels and environmental pollution issues, the electrolysis of water or organic hydrocarbons is a route which is widely investigated by considering fuel cell technology for clean and renewable energy capturing from solar energy via the photo sensitized electrolysis or heterogeneous electrolysis (PEC reactions). ${ }^{4-8}$ In particular, the PEC reactions (both cathodic and anodic), are controlled by many aspects and one of those key quantities is the redox level of reactants upon hetero-junction formation between redox couples, which is being subject of immense research interest in electrochemistry. ${ }^{9-13}$

Experimentally, the redox levels of materials are commonly measured through the flat-band measurements for standalone electrode materials. ${ }^{14-15}$ Theoretically, different approaches have been proposed consisting to define the band positions with respect (1) to vacuum (slab-vacuum approach) ${ }^{16-17}$, (2) to another solid (two-solids interface model) ${ }^{18}$, (3) to the transition level of hydrogen in a two-solids hetero-junction mode ${ }^{19}$, (4) or to a solid-liquid interface model. ${ }^{20-22}$ All these methods are, basically, concerned with finding of an intrinsic quantity of the modelled materials, which can be used to line-up their band-edges using a common energy scale. Finally, Greiner et al. in 2011 have proposed an experimental strategy using ultraviolet photo-luminescence measurements which uses the work-function and ionization potential energy allowing to realign the Highest Occupied and Lowest Unoccupied Molecular Orbitals (HOMO and LUMO) of well known insulating oxides ${ }^{12}$. The present investigation proposes a new approach allowing to define an absolute energy reference from first-principles calculation to compare the band-edge energies i.e. valence band top, $V_{b e}$ (so-called HOMO) and conduction band bottom, $C_{b e}$ (so-called LUMO) of 11 oxides and nonoxide semiconductors (photocatalysts). We also have discussed results with each other compounds compared to the Vacuum Energy Scale (or the Neutral Hydrogen Electrode (NHE)).

\section{METHODOLOGY}

Whatever the ab-initio technique, the zero of potentials in an infinite crystal is completely arbitrary. ${ }^{23}$ This is why all methods based on periodic boundary conditions cannot be used 
directly to compare the band positions of different solids. One way to overcome this limitation is to consider a semi-infinite crystal, i.e. boundary conditions not applied to the all three crystallographic directions. For instance, as soon as calculations are carried out on isolated blocks well separated from its image, a zero of potential (vacuum potential) can be defined and thus an absolute energy scale is achievable. A way to approximate this semi-infinite crystal is to create a slab model with a sufficiently large vacuum spacing in between the slab images. ${ }^{16-17}$ It will then mimic the existence of a surface with a zero of potentials which is defined in the centre of the vacuum region, if this region is large enough. ${ }^{24}$ While such a model has been widely used in the past, it appears to be highly dependent on the conditions of the calculations. In addition, the definition of the width of the vacuum length is not trivial at all and is material's dependent. Details of the methodology will be discussed in the following subsections step by step.

Choice of various computational parameters for the electronic properties simulations i.e. density of states for different slab models in DFT and hybrid-DFT level is given in the Supporting Information (SI) Section S1, which also include information on the use of plane-wave codes, kinetic energy cut-off, $k$-mesh, convergence criteria of energy as well as density and pseudopotentials. However, note that since we are dealing with the band extrema around the band-gap of bulk materials thus surface relaxation was not taken into account and slabs were formed using the experimental bulk crystal structures. The slabs were completely stoichiometric and having nonpolar termination (see SI Figure S1). Our argument is in direct line with the previous theoretical studies showing negligible impact of non-polar surface relaxations. ${ }^{25-26}$ The choice of higher level $G W$ approximations with vertex corrections ${ }^{27}$ is out of scope in the present study, since superior performances of hybrid functionals are observed for band-gap and ionization potentials calculations for insulators and their interfaces. ${ }^{28}$ To some extent the role of the dielectric screening and exchangecorrelation in a range-separated hybrid functional for such slabs, is partially discussed later in the Results section. The thickness of the slabs was made sure in such a way that, the central part of the slab material behaves like the pristine bulk material, which is based on the convergence of calculated valence bands and electrostatic potential of atoms at the central part of the slab. Thus, our supposed calculations of slab are large enough (in terms of the slab thickness and its image distance) to mimic bulk properties. The transferability of estimating density of states (DOS) is robust, since it is independent of the choice of another plane-wave full-potential code i.e. WIEN $2 \mathrm{~K}^{29}$, and thus validates our choice of the computational tools.

Here we present two strategies, allowing to access the absolute $V_{b e}$ and $C_{b e}$ positions of semiconductors, namely the "modified vacuum approach" and the "helium-slab approach". The generation of the slab models is a common feature of these two methodologies. For each compound, slab models have been created in such a way to insure the convergence of the vacuum energy, which implies to properly control the thicknesses of the atomic and vacuum layers. Our tests lead to define reasonable thicknesses of $c a$. $10 \AA$ and $20 \AA$ for the atomic and vacuum layers in a slab model, respectively. For instance, let's consider a model consisting of an isolated helium $(\mathrm{He})$ atom inside a vacuum layer (one Helium atom is placed at the central region of the vacuum of a slab-model), which separates image of $\mathrm{GaP}$ atomic layers (see SI Figure S1). The calculation of vacuum energy and its convergence is checked and illustrate in the SI Figure S2. Then, to get a moderate accuracy of the energy of the $\mathrm{He}\left(1 \mathrm{~s}^{2}\right)$ orbital energy, a vacuum thickness of $40 \AA$ is required, at least from ground state DFT level. However, in plane-wave approach the vacuum region is filled by plane-waves. For such a large vacuum region the price to pay is less significant in standard DFT (LDA or GGA) and becomes a real limitation using hybrid functionals. At PBE-GGA level, using a vacuum thickness of only $20 \AA$ leads to an error of $0.1 \mathrm{eV}$ in the estimation of the $1 \mathrm{~s}^{2}(\mathrm{He})$ orbital energy vs. Vacuum width for blende phase of GaP (see SI Figure S3). Similarly, the thickness of the material slab within the slab model was tested for these compounds, and one example for rutile $\mathrm{TiO}_{2}$ is give in SI Figure $\mathbf{S 4}$ i.e. the convergence of the average atomic core potential of slab vs. vacuum energy level.

Finally, it is discussed later the necessity of using a hybrid functional to estimate the influence of the hybrid mixing Hartree-Fock (HF) exact-exchange parameter, $\alpha$ using standard HSE06 functional from Heyd-Scuseria-Ernzerhof $f^{30-32}$ on the $a b$-initio energy positioning of band extrema. For clarity, $\alpha=0$ and $\alpha=1$ correspond to pure DFT and Hartree-Fock calculations, respectively. Replacing part of the DFT approximate exchange by part of the HF exact exchange leads to a linear evolution of the energies, as evidenced in SI Figure S5. Thus, the accuracy of our prediction was further improved by using post-DFT short-range corrected screened Coulomb hybrid functional calculations using Heyd-Scuseria-Ernzerhof hybrid functional HSE06 for given default screening parameter $(\mu)$ $0.2 \AA^{-1}$. The later parameter is actually separating the short and long range contribution of the Coulomb kernel, with Fockexchange $25 \%$ for short-range and neglected in the long-range part. The choice is based on the calculated standard enthalpies of molecular systems in $\mathrm{G} 2$ data-set $^{30}$, and extremely robust for predicting electronic properties of insulting and semiconductor solids. ${ }^{33-36}$ Based on these choices, we have developed (see below) an approach allowing to estimate errors in a systematic manner and corrected it during the post-treatment of the computed data as well as band extrema alignment. These corrections lead to reasonable accuracy of the prediction compared to their available flat-band measured data of $V_{b e}$ and hence $C_{b e}$ for these standalone semiconductors i.e. rutile and anatase $\mathrm{TiO}_{2}$, wurtzite $\mathrm{ZnO}$, rutile $\mathrm{SnO}_{2}$, blende $\mathrm{SiC}, \mathrm{GaP}$, GaAs, InP, ZnTe, CdS and CdSe., ${ }^{9,14}$

\subsection{Modified Vacuum Approach}

The first stage of the alignment technique is the "modified vacuum approach". To align the redox positions of band-gap in potential photocatalysts at the same referential, the valence band top edge, $V_{b e}$ is estimated and realigned from modified vacuum approach, and the method is illustrated in detail here considering the example case of some hypothetical semiconductor (See Figure 1). In the top panels a) and b) of Figure 1, the total DOS of bulk and corresponding slab model are shown, respectively. The $V_{b e}$ is taken to be $V_{b e}$ (bulk) = $E_{F}$ (bulk) and $V_{b e}($ slab $)=E_{F}\left(\right.$ slab); in both cases $E_{F}$ corresponds to the highest occupied state i.e. Fermi level with respect to their reference energy scale, $E_{R e f}$ (bulk) and $E_{R e f}$ (slab), in bulk and slab respectively. However, it is well 
known that the later reference quantities are completely arbitrary and instinct to the given first-principles calculations based on different basis set choice. Since, surface termination or polarity in a slab calculations can lead to an important role for defining the Fermi level, thus considering it directly as $V_{b e}$ could be erroneous. ${ }^{37}$ So, searching of proper surface pollution free $V_{b e}$ and then vacuum reference energy for realigning $V_{b e}$, led to us proposing the so-called modified vacuum approach, a method based on the core level shift of constituent atoms. It is worth to mention here that the modification on the standard vacuum-slab approach is hidden herein. More explicitly speaking, we use the calculated vacuum energy level as a reference from a slab model calculation, $E_{\text {vacuum }}($ slab), and we assume the first-ionization energy is $\left[E_{\text {vacuum }}(s l a b)-V_{b e}(s l a b)\right]$, which is slightly different from $\left[E_{\text {vacuum }}(\right.$ slab $)-E_{F}($ slab $\left.)\right]$, where surface induced pollution on the valence band top edge, $V_{b e}$ is excluded by neglecting the direct choice of Fermi level, $E_{F}$. For example, in case of the $\mathrm{GaP}$ or $\mathrm{TiO}_{2}$, we have considered the relative shift of $1 \mathrm{~s}^{2}$ core-states of these cations from their computed electrostatic core potentials of atoms in the pristine bulk and central region from a slab model calculation. Because, we assured sufficiently thicker slabs containing at least 15 atomically thin layers (see SI Figure S4), so that one can imagine the bulk region of the slab is equivalent to the infinite periodic bulk phase of the pristine material at least from computed electrostatic and electronic properties. More specifically, the average electrostatic core potential of bulk atoms of these two models will be same due to the similar volume per formula unit in the central part of the slab material. Thus, we have calculated the core level shift, using equation 1 (eq. 1), as given below:

$$
\Delta E_{\text {core }}=E_{\text {core }}(\text { slab })-E_{\text {core }}(\text { bulk })
$$

Inserting the core level shift, $\Delta E_{\text {core }}$ into the calculated DOS of the bulk phase, one can estimate the surface pollution free valence band top, so-called $V_{b e}$ from a standard slab model calculation, which is shown in the panel c) of Figure 1. This superposition procedure is quite reasonable way than the Fermi level shift, because from our estimation in case of rutile $\mathrm{TiO}_{2}$, for example surface influenced error $0.24 \mathrm{eV}$, which must be excluded from the estimation of $V_{b e}$, as soon as we are mimic to search for bulk phase band-edge realignment. We should mention here with caution that the given nomenclature core level shift differs from the already existing key word in experimental context i.e. surface core-level shift (SCLS or simply CLS) in photoelectron spectroscopy or microscopy measurements, despite their analogous usage.

Finally, in order to align the computed $V_{b e}$ we used the vacuum energy, $E_{\text {vacuum }}(s l a b)$ which is calculated from the slab model. Thus, the vacuum-level shift is considered with following eq. 2 ,

$$
\begin{gathered}
\Delta E_{\text {vacuum }}=E_{\text {vacuum }}(\text { absolute })-E_{\text {vacuum }}(\text { slab }) \\
=-E_{\text {vacuum }}(\text { slab })
\end{gathered}
$$

Since, the absolute vacuum energy is $0.00 \mathrm{eV}$ by definition (cf. Figure 1.d), the calculated vacuum level for slab was finally set to zero energy.

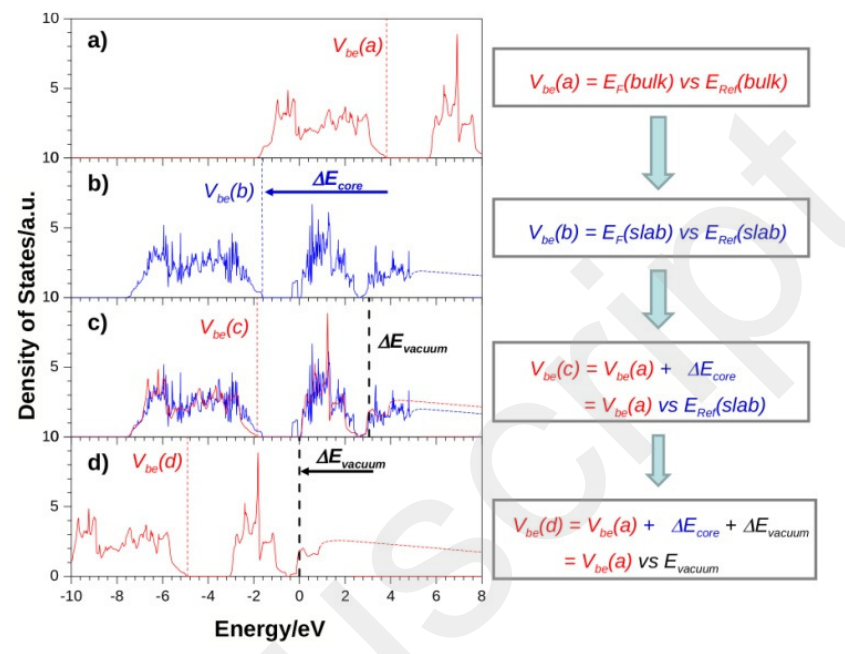

Figure 1. The schematic representation of the computational methodology to predict of valence band top, $V_{b e}$ considering some example hypothetical semiconductor. In the panel a) and b), the total DOS of the bulk and slab are shown, respectively and their $V_{b e}$ are taken as corresponding calculated Fermi levels. Panel c), they are superimposed based on the average core energy level shift of constituent atoms $\left(\Delta E_{\text {core }}\right)$ shift. Whereas, in the panel d) $V_{b e}$ is shown excluding the surface impurities and rescaled versus vacuum level shift $\left(\Delta E_{\text {vacuum }}\right)$.

In a similar strategy, the $V_{b e}$ is predicted for rest of the other slab models, and hence so-called modified vacuum approach is described herein. However, the calculated vacuum energy level in the current approach $E_{\text {vacuum }}(s l a b)$ is sum of the local potential summation technique by including the ionic, Hartree and exchange-correlation potentials, which mimics to be a true reference due to long range Coulomb potential. Hence, it is eventually affected by the Coulomb interactions between image charges imposed by the periodic boundary conditions. Thus, calculated vacuum energy level from slab models is pseudo in nature, inheritably polluted within periodic boundary conditions in first-principles plane-wave calculations. Thus, novelty of our work is not only the modified vacuum approach, but also correcting the error on the reference vacuum energy level, $E_{\text {vacuum }}($ slab) via helium-slab approach. This has been discussed and the problem is described in the next subsection.

\subsection{Helium Atom in the "Modified Vacuum Approach"}

The second and final stage of the current methodology is the "helium-slab approach". As we saw, considering a finite size vacuum length along the surface grown direction, spontaneously accounts an amount of error in the estimated vacuum energy level, thus additional improvement must be needed. This is obviously telling us a necessity of a probe that can help us to eliminate the accounted error on the vacuum energy reference. The choice of such probe is the isolated Helium 
(He) atom, a noble and unreactive gas element. The probe should be such that, 1) it has to be smaller in volume, which allows one to use relatively smaller supercell size for computation, 2) the chemical inertness of the probe would not provoke to have any stronger interactions with neighboring slab layers or image of itself due to the imposed periodic boundary conditions, 3) the active energy level (here $1 \mathrm{~s}^{2}$ of $\mathrm{He}$ ) of the probe should be far below in the energy scale than the redox level or valence band hybridization width of these insulating solids i.e. probe is highly unreactive. The difference of the current approach than the previously mentioned modified vacuum approach is essentially due to the fact that the energy level of $\mathrm{He}\left(1 \mathrm{~s}^{2}\right), E_{H e}$ as the reference energy for correcting further the vacuum energy level computed from the standard slab model calculations and hence the so-called helium-slab approach (or He-Slab approach) is proposed, herein.

For an isolated $\mathrm{He}$ atom, experimentally the first-ionization energy is $+24.59 \mathrm{eV}$ i.e. the $\mathrm{He}\left(1 \mathrm{~s}^{2}\right)$ level is located at the $+24.59 \mathrm{eV}$ below the absolute vacuum, which is quite lower than presently used semiconductor's $V_{b e}$ or $C_{b e}$. However, the correct description of the $\mathrm{He}\left(1 \mathrm{~s}^{2}\right)$ energy level from ground state DFT is matter of question, which should be figured out in the initial step, despite the limitations of atomization energy or other chemical properties calculation from DFT. In all hereafter discussed He-slab models, the average value of $\mathrm{He}\left(1 \mathrm{~s}^{2}\right)$ state is calculated using PBE-GGA functional (Hartree-Fock exact exchange $\alpha=0.00),-15.64 \mathrm{eV}$ i.e. $36 \%$ smaller than the experimental value. In case of the $\mathrm{GaP} \mathrm{He}-\mathrm{Slab}$ model, $\alpha$ dependence of the $\mathrm{He}\left(1 \mathrm{~s}^{2}\right)$ energy state and the core potential is shown for four different value of $\alpha=0.00,0.20,0.25,0.73$ in the SI Figure S5. Thus, we conclude from these following checks that significant amount of the Hartree-Fock exact exchange must be added to better describe the $\mathrm{He}\left(1 \mathrm{~s}^{2}\right)$ energy state, $E_{H e}$ to get closer into experimentally known data. Considering, a linear extrapolation up to $\alpha=1.00$ from all He-Slab models based on their calculated values at $\alpha=0.00$ and 0.25 using HSE06 functional, the average value of $E_{H e}$ is estimated to be $-23.86 \mathrm{eV}$ (at $\alpha=1.00$ ). However, this is little bit off $(\sim 2.9 \%)$ but quite closest to the experimental one i.e. -24.59 $\mathrm{eV}$ (See SI Figure S6) within the present theoretical limit. We will also see below in the Results section that such approach using He-Slab model will help us to reduce the mean absolute error (MAE) or standard deviation (SD) of our predictions. These statistical quantities MAE and SD define the quality of the predicted $V_{b e}$ versus the experimental data for a set of $N$ number of compounds. However, we must mention with caution that limitation of computation models, MAE cannot be directly linked to the experimental errors in the measured $V_{b e}$, despite their analogous applications. In the practical exp. the error accounts from instrumental set-up or other chemical and physical environments of each compounds, whereas MAE is an absolute, average statistical error bar from the chosen computational set-up. On the other hand, the later SD defines the dispersion of the error around the calculated MAE.

As mentioned previously, the $\mathrm{He}\left(1 \mathrm{~s}^{2}\right)$ energy level plays as a probe in the slab model and help us to correct the calculated vacuum energy level from each separately modeled materials. Such estimation is possible by considering the helium gas phase within a plane-wave periodic potential based calculations using DFT. In the SI Figure S7, the evolution of the $\mathrm{He}\left(1 \mathrm{~s}^{2}\right)$ energy with the cell size i.e. the He-He distance $(d)$, is shown from PBE-GGA $(\alpha=0.00)$ and HSE06 $(\alpha=0.25)$ functional calculated data. It is clearly seen that the convergence of $E_{H e}$ reaches for the cell dimension $(d)$ greater than $d$ $=30 \AA$ with a calculated value $-15.71 \mathrm{eV}$, compared to the previously mentioned average value from all eleven He-Slab models, which was $-15.64 \mathrm{eV}$ at PBE-GGA level. Thus, an amount of error due to the He image charge interactions is $0.08 \mathrm{eV}$, and essential to account for correcting the $E_{\mathrm{He}}$ energy core state $1 \mathrm{~s}^{2}$. Also, due to the different chemical neighbors of $\mathrm{He}$ atom in different He-slab models, it does not have same chemical inertness and hence the errors are different. Within the PBE-GGA or HSE06 calculated data, using Lorentz fit, an error amount was evaluated for all cases and these values are eliminated from the prediction (see SI Table S2). It should be noted that this amount is small (few tens of meV) and shifted in the same direction, hence the approach is robust and arises fidelity for application. In the next sections, results from our theoretical methodologies are discussed.

\section{Results and Discussions}

The section is followed by three subsections, based on the results from two afore mentioned methodologies along with their implication in photocatalysis or in broader extent into electrochemistry. Also, as anticipated earlier, here results are shown for rutile- and anatase $\mathrm{TiO}_{2}$, wurtzite $\mathrm{ZnO}$, rutile $\mathrm{SnO}_{2}$, blende phase of GaP, GaAs, InP, ZnTe, CdS, CdSe, and SiC, those are well known and qualitatively important for catalytic based device applications or in optoelectronics using their standalone and/or hetero-junctions.

\subsection{Modified Vacuum Approach}

We have done prediction based on the available experimental data from flat-band measured $V_{b e}$ of these eleven insulating solids (except $\mathrm{ZnTe}$ ). ${ }^{38}$ The calculated values of $V_{b e}$ as predicted following the eq. 1 and eq. 2, referring to the schematics shown in Figure $\mathbf{1}$ and corresponding numerical data is shown in Table 1. Alongside the calculated data, the experimental data against NHE at neutral $\mathrm{pH}$ and room temperature or Vacuum scale reference are also tabulated (see Table 1) ${ }^{9,14}$. Our prediction for $V_{b e}$ were done from the HSE calculations using both $\alpha=0.00$ and 0.25 for Hartree-Fock exact exchange. It is clear from the Table 1 that at the PBE-GGA $(\alpha=0.00)$ level, predicted data set is far from the experimental values with MAE $=1.4 \mathrm{eV}$ and $\mathrm{SD}=0.88 \mathrm{eV}$. With the increasing value of $\alpha=0.25$ within the HSE06 functional, these two error bars were reduced to 0.84 and $0.64 \mathrm{eV}$, respectively. Thus, we considered all the linear variations of these quantities needed to estimate the theoretical value of $V_{b e}$ (see SI Figure S5) based on the input from the PBE-GGA and HSE06 calculated data, using the least square-fit procedure on experimental data (known flat-band measured values of $V_{b e}$ ) and one degree of freedom ( $\alpha$ value). We have obtained the possible better agreement at $\alpha=0.635$ from this modified vacuum approach. The graphical representation of these data using $\alpha=0.635$ is shown in Figure 2. Out of all these cases, we have seen that the calculated $V_{b e}$ is moving towards the experimental values at the given $\alpha=63.5 \%$ which gives us the best fit assigned from the calculated MAE and SD respectively, 0.33 and 0.43 eV (cf. Table 1).

Essentially, the limitation of the dielectric screening or vacuum width dependent dielectric function in slab calculation 
forbids us optimizing the Fock-exchange for these compounds from dielectric depend self-consistent runs from plane-wave based tools. ${ }^{39}$ Thus linear variations with least-square fitting would be another safe approach. However, we must mention here that the choice of the Hartree-Fock exchange is thus, not purely from the empirical choice from the standard hybrid functional, but more likely semi-empirical, as referred to the known experimental flat-band data. Despite optimizing the screening parameter in HSE06 functional, ${ }^{40}$ we propose optimized Fock-exchange in a short range-separated screened Coulomb functional along with given screening parameter 0.2 $\AA^{-1}$. Also, the current method proposes a way to realign the valence and conduction bands of semiconductors in relative manner, other than predicting the band-gaps i.e. the difference of two band extrema at high symmetry $k$-point of first Brillouin zone. So, results are discussed and elaborated regarding to their relative valence and hence conduction bands location in an electrochemical reference energy scale, which would be interest for broader audiences in theoretical developments as well as in electrochemistry research domains.

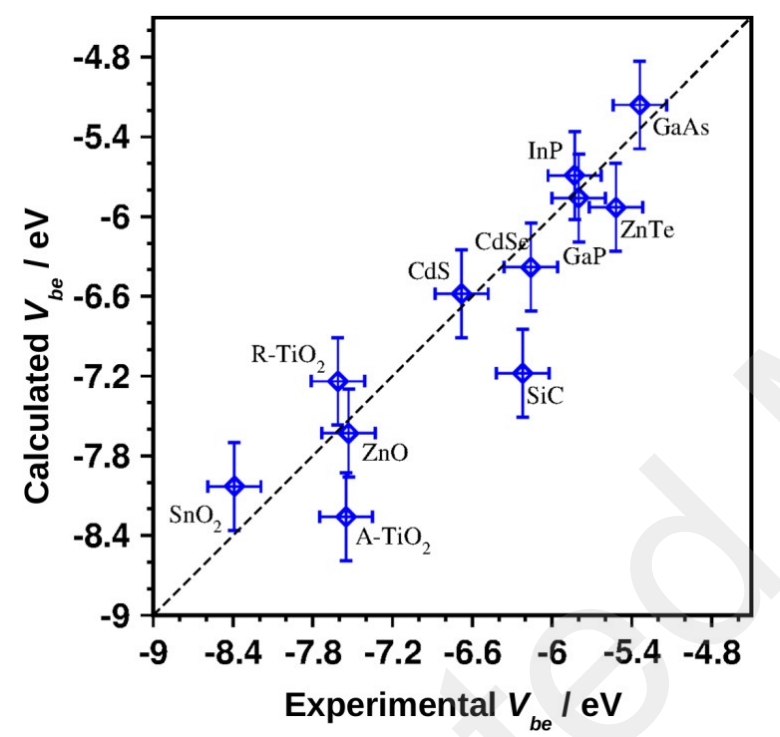

Figure 2. The predicted values of $V_{b e}$ using the "Modified Vacuum Approach" as estimated from HSE calculations with HartreeFock exchange $\alpha=63.5 \%$. The error bars from experimental $(0.2 \mathrm{eV})$ and theoretical MAE $(0.33 \mathrm{eV})$ are taken into account.

Looking at Figure 2, the solid black dashed line passes through the diagonal of the square plot is the measure of best accuracy of our predictions compared to the experimental data. The maximum underestimation of the $V_{b e}$ was found for $\mathrm{SiC}$ compound, which is about $0.96 \mathrm{eV}$, follows by $\mathrm{A}-\mathrm{TiO}_{2}$, $0.71 \mathrm{eV}$. An overestimation of about $0.40 \mathrm{eV}$ was found for the rutile $\mathrm{TiO}_{2}$ and $\mathrm{SnO}_{2}$ at the given Fock-exchange $63.5 \%$. These error amounts are reduced once the He-Slab approach is used, where the impact of true vacuum reference energy was estimated which is free from plane-wave induced Coulomb interactions and results are discussed in next subsection.

\subsection{He-Slab Approach}

Use of higher $\alpha$ value within the modified vacuum approach was obviously not enough reasoning, since MAE or SD are quite larger than the experimental error bar limit 0.10-0.20 eV. Thus, incorporation of error from the image charge interactions in a standard slab-model is very much crucial here i.e. inclusion of vacuum energy corrections to remove the pseudo behavior of material dependent vacuum energy level. The Fock-exchange was predicted from least square-fit here to be $\alpha$ $=73 \%$. Using $\alpha=0.73$ in a HSE06 calculations, the computed values are reported for $V_{b e}$ along with PBE-GGA $(\alpha=0.00)$ and standard HSE06 $(\alpha=0.25)$ functionals. Then, presently predicted numerical values of valence band tops, $V_{b e}$ are also given through the Table 2.

As we can see, the errors are reduced with estimated MAE is nearly $0.20 \mathrm{eV}$ and $\mathrm{SD}=0.27 \mathrm{eV}$ at $\alpha=73 \%$ (see details in Table 2), those are in excellent agreement with the available experimental data, till date. ${ }^{9.14}$ The plotting of the predicted $V_{b e}$ at $\alpha=0.73$, against their experimental values are shown in Figure 3. As similar to the previous plot (cf. Figure 2), here also the error bars from experimental $(0.2 \mathrm{eV})$ and theoretical MAE $(0.20 \mathrm{eV})$ are taken into account. The maximum underestimation is found for the $\mathrm{A}-\mathrm{TiO}_{2}$ and $\mathrm{GaP}$ compound which is about $0.30 \mathrm{eV}$. However, for the blende $\mathrm{ZnTe}$, we could not find a clear flat-band measurement, thus peaked up the photoemission data of valence and conduction band off-set of $\mathrm{CdSe} / \mathrm{ZnTe}$ interfaces from the experimental literature. ${ }^{38}$

Hence, we have corrected the vacuum reference energy from first-principles calculations from slab-model approach and proposed an absolute vacuum energy level, which is applied to predict the $V_{b e}$ of photocatalysts in a common referential. In further extent, the methodology is rationalized from the prediction procedure of both the valence band top $V_{b e}$ and conduction band bottom $C_{b e}$ two possible solutions are proposed here.

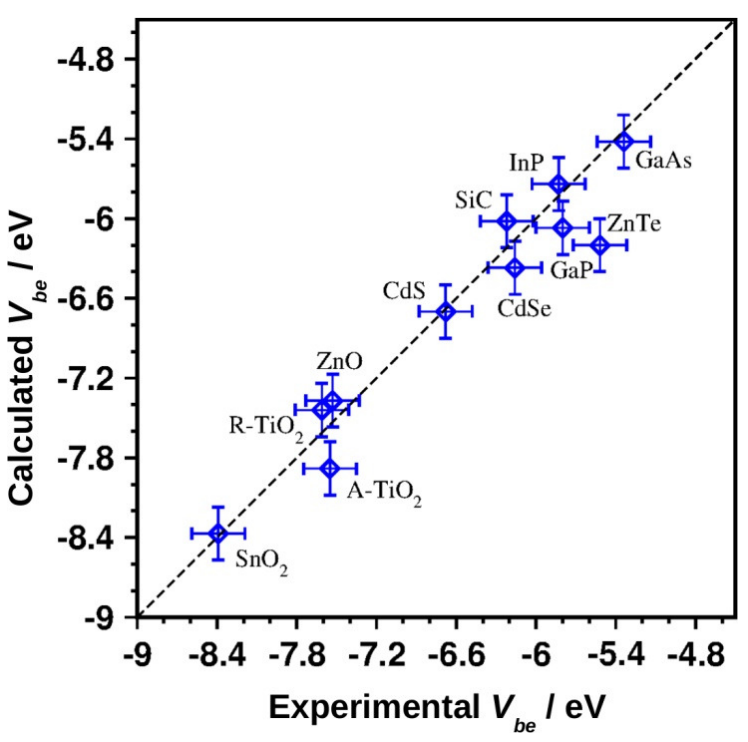

Figure 3. The predicted values of $V_{b e}$ using our "He-Slab Approach" as estimated from HSE calculations with Hartree-Fock exchange $\alpha=73 \%$. In both axis error bar is $0.2 \mathrm{eV}$.

Either, by adding the known experimental band-gap to the predicted $V_{b e}$ values, and hence get $C_{b e}$ as similar fashion to 
the flat-band measurement technique or otherwise use postDFT hybrid functionals or many-body corrections to get reasonable band-gap of test compounds to estimate the $C_{b e}$ in complete first-principles approach. The later might be issue of future research, despite the issue of correct band-gap calculation of solids from hybrid density functional theory. However, using the first strategy, the redox position realignments are shown in Figure 4, with respect to water oxidation and reduction level vs. NHE or Vacuum Scale. Quite reasonable agreement is found for all these calculated $V_{b e}$ and hence $C_{b e}$ levels compared to their experimental data. More physical and chemical implications of these predictions are discussed in the next subsection.

\subsection{Outlook and Electrochemical Implications}

Looking at Figure 4, we can clearly see the qualitative trend is obviously good for all these cases, as compared to their experimental data from Nozik, 1978 or Grätzel, 2001.9,14 Specially, if we look to the general trend of these $C_{b e}$, for rutile $\mathrm{SnO}_{2}$, $\mathrm{TiO}_{2}$ and wurtzite $\mathrm{ZnO}$, they are very much systematically aligned from our computational experiments. In particular, $C_{b e}$ is moved gradually into higher in energy, similar to their known experimental data. Also, for other non-oxides i.e. CdS vs. CdSe and InP vs. GaAs pairs, predicted $C_{b e}$ are also well agreed with their experimental data, and quite rationally. However, prediction of the relative $V_{b e}$ position for two oxides i.e. rutile and anatase $\mathrm{TiO}_{2}$ are contradicting with the existing experimental flat-band data from Kavan et al. $1996 .^{41}$ It says that the band off-set of $V_{b e}$ to be $0.2 \mathrm{eV}$ more positive for anatase than rutile. Indeed, this could be due to the presence of impurities (trace of unavoidable presence of metals i.e. $0.22 \%$ $\mathrm{Al}, \mathrm{V}$, or $\mathrm{Zr}$ ) in the anatase sample during synthesis, as well as practical surface impact absent in our theoretical models. As discussed earlier in an ideal situation, we have minimized the surface effects from the chosen (110) or (010) slab models for rutile or anatase respectively, compared to the experimental impedance analysis were done for (001) and (101) surfaces, respectively.

On the other hand, recent work from Scanlon and co-authors, 2013 proved the relative $V_{b e}$ off-set $0.4 \mathrm{eV}$ higher in energy and $0.2-0.3 \mathrm{eV}$ off-set for $C_{b e}$ higher in energy for rutile and anatase from core-shell nanoparticles. ${ }^{42}$ They have used bandedge alignment of these two oxides very rigorously from the experimental photo-emission, atomic core-potential alignment and Quantum-mechanical combined Molecular-mechanical DFT computations. Indeed, considering the experimental band-gap 3.03 and $3.20 \mathrm{eV}$ respectively for rutile and anatase, it lead to $\sim 0.2-0.3 \mathrm{eV}$ higher energy off-set of $C_{b e}$ and also $V_{b e}$ off-set $\sim 0.4 \mathrm{eV}$ higerer in energy for rutitle than anatase. This is absolutely similar in trend to the prediction from Scanlon et al. 2013 and thus validated the robustness of our current theoretical methodology. Also we observed that the qualitative location of $V_{b e}$ band off-set of the rutile $\mathrm{TiO}_{2}$ and $\mathrm{ZnO}$ have also been obtained with similar trend than known exp. $0.14 \mathrm{eV}$ versus our prediction $\sim 0.07 \mathrm{eV}$ which is higher for $\mathrm{ZnO}$ than rutile $\mathrm{TiO}_{2}{ }^{42-43}$ Further theoretical and experimental researches are needed to correlate the redox mechanism involved with the flat-band measurement of solid electrodes and photoemission spectroscopy study of the heterogeneous solid-liquid interface, a possible approach of dealing with the $\mathrm{pH}$ dependence through the solvents and/or electrolytes.
Thus, a computationally cost effective output from our theoretical methodology might be helpful to the community, working on the photoelectrochemical, electrolysis domain either using theoretical tools or in some extent it would be helpful to control experimental design and measurements. By inventing such corrected vacuum energy strategy, absolute reference energy is proposed in similar footling like experimental one, which can be helpful for further development of redox reaction based energy storage and conversion technologies in a cost effective and efficient manner. Specifically, the goodness of the He-slab approach is indirectly measured through the statistical quantities MAE and SD are 0.2 and $0.27 \mathrm{eV}$, respectively equivalent to the experimental real error bar $0.1-0.2 \mathrm{eV}$ for these bulk photocatalysts.

It should be noted here that the current methodology does not necessarily take into account the actual description of the real surface. Truncation in any orientation at any position of the material is sufficient, even though it is not an energetically stable surface, but at the cost of computational difficulties. Thus, non-polar surface or slab model choice was one of the simplest approximations. The major problem concerning on the down-folding and up-folding of bands at the surface depending on the materials, quasi-2D types of the solids and the solvent's dielectric nature are especially could be grand future challenges to improve the current methodology. Hence, we embark our conclusion with caution that in our present approach even though we did not take into account in our computations all correct experimental variables, including the temperature, pressure or $\mathrm{pH}$ to reach into the reasonable accurate description of the $\mathrm{H}^{*}$ or $\mathrm{OH}^{*}$-intermediated radicals or hydrocarbon adsorption, which are controlled by the relative redox of the electrodes, but results are expected to be acceptable in broader sense and rationally, as soon as we are looking for valence and conduction band edges of bulk solid photocatalysts.

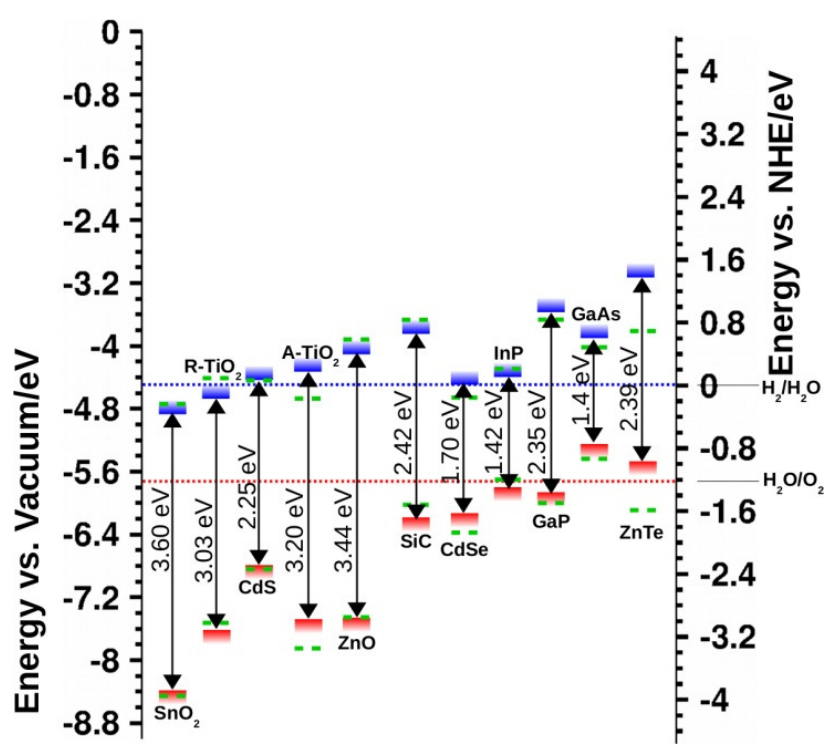

Figure 4. Results from He-Slab Approach. The calculated $V_{b e}$ and predicted $C_{b e}$ at $\alpha=73 \%$ (green dashed bars) are represented 
with the available experimental data (red and blue solid bars, respectively), using Vacuum (left) or NHE (right) energy scale. Values of $C_{b e}$ were estimated by adding the experimental bandgap for each compounds with calculated $V_{b e}$. The two dotted lines are the reduction (blue) and oxidation (red) redox level of $\mathrm{H}_{2} \mathrm{O}$. Available experimental flat-band data are taken at $\mathrm{pH}$ neutral and room temperature.

\section{Conclusions}

In conclusion, the present theoretical strategy which is used to calculate and predict realignment of the valence band top for a set of different oxides and non-oxides bulk materials within a reasonable accuracy from lowest computational cost and same referential, is achieved reasonably. Despite the semi-empirical treatment of the Fock-exchange, the core level shift within the "modified vacuum approach" removes the surface pollution from the surface induced property the HOMO energy level or so-called valence band top. Then, within the He-Slab approach, the $\mathrm{He}$ atom helps to correct the material dependent vacuum energy level. Indeed, the $\mathrm{He}\left(1 \mathrm{~s}^{2}\right)$ energy level was used to find the absolute vacuum level, and validating the artiness of the present first-principles calculations applied for redox realignment for some important inorganic bulk photocatalysts. In fact, the main reason why we have reached such to an excellent accuracy of the predictions, having MAE $\sim 0.2 \mathrm{eV}$, resides well within the He-slab model choice. However, our method does not explicitly have treated the $\mathrm{pH}$, thus limits the approach to be applied in the extreme realities of electrochemistry in practical lab set-up. With this caution, we conclude that our ultimate goal is to find absolute reference energy scale for solid state compounds from computational experiments, have been qualitatively achieved here. We are expecting such study would be helpful in future development and progress using redox reactions based different technological applications i.e. electro/photo-catalysis, fuel-cell, and photovoltaics based solar energy conversion and storage issues. Finally, our calculations are done from independentparticle DFT approach excluding correct excited state properties of solids involving excitons, which will be matter of future research interest to get further improvement of the current theoretical methodology in similar proximity that includes photon excitations.

Indeed, computations based on the 2D periodic boundary conditions and atomic orbitals based on Gaussian type basissets would be also further interest. Recently, it has been claimed by Pacchioni and co-workers ${ }^{44}$ the novelty of using localized basis sets based calculations i.e. relatively more cheaper computation time to get band-gap and dielectric constant for insulating bulk solid oxides (non-magnetic) and layered compounds (van der Waals) using the B3LYP and Coulomb Attenuation method based long-range corrected version of B3LYP hybrid functional, CAM-B3LYP respectively. Also, impact of the solvation energy as proposed for solid-liquid interfaces ${ }^{45-47}$ in solvation energy corrected density functional theory will be incorporated in future studies.

\section{ASSOCIATED CONTENT}

Supporting Information: This material is available free of charge via the Internet at http://pubs.acs.org. Briefly Table of Contents as follows:

Section S1: Computational Details; Section S2: He-Slab Models Section S3: Dependence of Vacuum Width vs. DFT Functional; Section S4: Energy of $\mathrm{He}\left(1 \mathrm{~s}^{2}\right)$ vs. Vacuum Width Check; Section S5: Layer Thickness vs. Vacuum Width Check; Section S6: Atomic Core Potential vs. Exact-exchange in DFT Functional; Section S7: Energy of $\mathrm{He}\left(1 \mathrm{~s}^{2}\right)$ vs. Exact-exchange of DFT Functional; Section S8: Energy of $\mathrm{He}\left(1 \mathrm{~s}^{2}\right)$ vs. He-He Interaction Distance in Model

\section{AUTHOR INFORMATION}

\section{Corresponding Authors}

*Tilak Das,

E-mail : tilak.das@unimib.it

*Xavier Rocquefelte,

E-mail: xavier.rocquefelte@univ-rennes1.fr

\section{Present Addresses}

${ }^{\dagger}$ Current Address: Dipartimento di Scienza dei Materiali, Università degli Studi Milano-Bicocca, Via Roberto Cozzi 55, Milano 20125, Italy;

E-mail: tilak.das@unimib.it

${ }^{*}$ Current Address: Institut des Sciences Chimiques de Rennes UMR 6226, Université de Rennes 1, Campus de Beaulieu, 35042 Rennes, France;

E-mail: xavier.rocquefelte@univ-rennes1.fr

\section{Author Contributions}

All authors together had planned the scientific problem. Scientific strategies were developed by TD and XR to design the calculations. TD and XR did all calculations and data analysis. All authors wrote the manuscript, consulting with each other.

\section{ACKNOWLEDGMENT}

TD and XR would like to acknowledge computational resource used from laboratory Institut des Matériaux Jean Rouxel, Nantes (IMN) and CCIPL (Nantes) Computing Centre.

\section{ABBREVIATIONS}

DFT: Density Functional Theory; HOMO: Highest Occupied Molecular Orbital; LUMO: Lowest Unoccupied Molecular Orbital; $V_{b e}$ : Valence Band Top; $C_{b e}$ : Conduction Band Bottom; MAE: Mean Absolute Error; SD: Standard Deviation;

\section{REFERENCES}

(1) Franciosi, A; Van de Walle, C. G. Heterojunction band offset engineering. Surf. Sci. Reports 1996, 25, 1-140.

(2) Schlom, D. G.; Haeni, J. H. A Thermodynamic Approach to Selecting Alternative Gate Dielectrics. MRS Bull. 2002, 27, 198-204. 
(3) Dean, C.; Young, A. F.; Wang, L.; Meric, I.; Lee, G. -H.; Watanabe, K.; Taniguchi, T.; Shepard, K.; Kim, P.; Hone, J. Graphene based heterostructures, Solid State Commun. 2012, 152, 1275-1282.

(4) Osterloh, F. E.; Parkinson, B. A. Recent development in solar water-splitting photocatalysis. MRS BULLETIN 2011, 36(1), 17-22.

(5) Maeda, K.; Domen, K. Oxynitride materials for solar water splitting. MRS BULLETIN 2011, 36 (1), 25-31.

(6) Kudo, A. Z-scheme photocatalyst systems for water splitting under visible light irradiation. MRS BULLETIN 2011, 36 (1), 32-38.

(7) Zhang, J. Z. Metal oxide nanomaterials for solar hydrogen generation from photoelectrochemical water splitting. MRS BULLETIN 2011, 36 (1), 48-55.

(8) Kaneko, M.; Okura, I. Photocatalysis: science and technology, Springer-Verlag Berlin Heidelberg, New York, 2002, ISBN 3540-43473-9.

(9) Nojik, A. J. Photoelectrochemistry: Applications to solar energy conversion. Ann. Rev. Phys. Chem. 1978, 29, 189-222.

(10) Osterloh, F. E. Inorganic Materials as Catalysts for Photochemical Water Splitting. Chem. Mater. 2008, 20, 35-54.

(11) Zheng, Y.; Jian, Y.; Jaroniec, M.; Qiao, S. Z. Advancing the Electrochemistry of the Hydrogen-Evolution Reaction through Combining Experiment and Theory. Angew. Chem. Int. Ed. 2015, 53, 52-65.

(12) Greiner, M. T.; Helandar, G.G.; Tang, W. -M.; Wang, Z. -B; Qiu, J.; Lu, Z. -H. Universal energy-level alignment of molecules over metal oxides. Nat. Mater. 2011, NMAT3159.

(13) Tokito, S.; Noda, K.; Taga, Y. Metal oxides as a hole-injecting layer for organic electroluminescent device. J. Phys. D: Appl. Phys. 1996, 29, 2750-2753.

(14) Grätzel, M. Photoelectrochemical cells. Nature 2001, 414, 338344.

(15) Walter, M. G.; Warren, E. L.; McKone, J. R.; Boettcher, S. W.; Mi, Q.; Santori, E. A.; Lewis, N. S. Solar Water Splitting Cells. Chem. Rev. 2010, 110, 6446-6473.

(16) Frensley, W. H.; Kroemer, H. Theory of the energy-band lineup at an abrupt semiconductor heterojunction. Phys. Rev. B 1977, 16(6), 2642-2652.

(17) Van Vechten, J. A. Ionization potentials, electron affinities, and band offsets. J. Vac. Sci. Technol. B 1985, 3, 1240.

(18) Van de Walle, C. G.; Martin, R. M. Theoretical study of band offsets at semiconductor interfaces. Phys. Rev. B 1987, 35(15), 8154-8165.

(19) Van de Walle, C. G.; Neugebauer, J. Universal alignment of hydrogen levels in semiconductor, insulators and solutions. $\mathrm{Na}$ ture 2003, 423, 626-628.

(20) Angelis, F. D.; Fantacci, S.; Selloni, A. Alignment of dye's molecular levels with the $\mathrm{TiO}(2)$ band edges in dye-sensitized solar cells: A DFT-TDFT stydy. Nanotechnology 2008, 19, 424002.

(21) Wu, Y.; Chan, M. K. Y.; Ceder, G. Prediction of semiconductor band edge positions in aqueous environments from firstprinciples. Phys. Rev. B 2011, 84, 235301.

(22) Pham, T. A.; Ping, Y.; Galli, G. Modelling heterogenous interfaces for solar water splitting. Nat. Mater. 2017, 16, 401-408.

(23) Kleinman, L. Comment on the average potential of a Wigner solid. Phys. Rev. B 1981, 24, 7412.

(24) Choe, D.-H.; West, D.; Zhang, S. Band Alignment and the Built-in Potential of Solids. Phys. Rev. Lett. 2018, 121, 196.802.

(25) Jiang, H.; Shen, Y.-C. Ionization potentials of semiconductors from first-principles. J. Chem. Phys. 2013, 139, 164114.

(26) Stevanvić, V.; Lany, S.; Ginley, D. S.; Tumas, W.; Zunger, X. Accessing the capability of semiconductors to slit water using ionization potentials and electron affinities only. Phys. Chem. Chem. Phys. 2014, 16, 3706.
(27) Grüneis, A.; Kresse, G.; Hinuma, Y.; Oba, F. Ionization Potentials of Solids: The Importance of Vertex Corrections. Phys. Rev. Lett. 2014, 112, 096401.

(28) Hinuma, Y.; Kumagai, Y.; Tanaka, I.; Oba, F. Band alignment of semiconductors and insulators using dielectric-dependent hybrid functionals: Toward high-throughput evaluation. Phys. Rev. B 2017, 95, 075302.

(29) Blaha, P.; Schwarz, K.; Madsen, G. K. H.; Kvasnicka, D.; Luitz, J. WIEN2K (Eds. K. Schwarz), Technische Universitiät Wien, Vienna, 2001.

(30) Heyd, J.; Scuseria, G. E.; Ernzerhof, M. Hybrid functionals based on the screened Coulomb potentials. J. Chem. Phys. 2003, $118,8207$.

(31) Heyd, J.; Scuseria, G. E.; Ernzerhof, M. Influence of the exchange screening parameter on the performance of the screened hybrid functionals. J. Chem. Phys. 2006, 124, 219906.

(32) Paier, J.; Marsman, M.; Hummer, K.; Kresse, G.; Gerber, I.C.; Ángyán, J. G. Screened hybrid functionals applied to solids. J. Chem. Phys. 2006, 124, 154709; J. Chem. Phys. 2006, 125, 249901.

(33) Garza, A. J.; Scuseria, G. E. Predicting band gaps with hybrid functionals. Phys. Chem. Lett. 2016, 7, 4165-4170.

(34) Hinuma, Y.; Grünies, A.; Kresse, G.; Oba, F. Band alignment of semiconductors from density functional theory and manybody perturbation theory. Phys. Rev. B 2014, 90, 155405.

(35) Harrison, W. A. Electronic Structure and the Properties of Solids, (Freeman, San Francisco, 1980), p. 253.

(36) Rossmeisl, J.; Qu, Z.-W.; Zhu, H.; Kroes, G.-J.; Nørskov, J. K. Electrolysis of water on oxide surfaces. J. Electroanal. Chem. 2007, 607, 83-89.

(37) Yoo, S.-H.; Tadorova, M.; Neugebauer, J. Selective Solventinduced Stabilization of Polar Oxide Surfaces in an Electrochemical Environment. Phys. Rev. Lett. 2018, 120, 066101.

(38) Yu, E. T.; Phillips, M. C.; McCaldin, J. O.; McGill, T. C. Measurement of the CdSe/ZnTe valence band offset by X-ray photoelectron spectroscopy. J. Vac. Sci. Technol. B 1991, 9, 2233.

(39) Tian, T.; Scullion, D.; Hughes, D.; Li, L. H.; Shih, C.-J.; Coleman, J.; Chhowalla, M.; Santos, E. J. G. Electronic polarizability as the fundamental variable in the dielectric properties of the two-dimensional materials. Nano Lett. 2020, 20(2), 841-851.

(40) Chen, W.; Miceli, G.; Rignanese, G.-M.; Pasquarello, A. Nonempirical dielectric-dependent hybrid functional with range separation for semiconductors and insulators. Phys. Rev. Mater. 2018, 2, 073803.

(41) Kavan, L.; Grätzel, M.; Gilbert, S.E.; Klemenz, C.; Scheel, H. J. Electrochemical and Photoelectrochemical Investigation of Single crystal Anatase. J. Am. Chem. Soc. 1996, 118(28), 67166723.

(42) Scanlon, D. O.; Dunnill, C. W.; Buckeridge, J.; Shevlin, S. A.; Logsdail, A.L.; Woodley, S. M.; Catlow, C. R. A.; Powell, M. J.; Palgrave, R. G.; Parkin, I. P.; Watson, G. W.; Keal, T. W.; Sherwood, P.; Walsh, A.; Sokol, A. A. Band alignment of rutile and anatase $\mathrm{TiO}_{2}$. Nat. Mater. 2013, 12, 798-801.

(43) Wang, J.; Liu, X.-L.; Yang, A.-L.; Zheng, G.-L.; Wang, S.-Y.; Wei, H.-Y.; Zhu, Q.-S.; Wang, Z.-G. Measurement of wurtzite $\mathrm{ZnO} /$ rutile $\mathrm{TiO} 2$ heterojunction band offsets by X-ray photoelectron spectroscopy. Appl. Phys. A 2011, 103, 1099-1103.

(44) Das, T.; Di Liberto, G.; Tosoni, S.; Pacchioni, G. Band Gap of 3D Metal Oxides and Quasi-2D Materials from Hybrid Density Functional Theory: Are Dielectric-dependent Functionals Superior? J. Chem. Theory Comput. 2019, 15, 6294-6312.

(45) Andzelm, J.; Kölmer, C. Incorporation of solvent effects into density functional calculations of molecular energies and geometries. J. Chem. Phys. 1995, 103(21), 9312.

(46) Mathew, K.; Sundararaman, R.; Letchworth-Weaver, K.; Arias, T. A.; Hennig, R. G. Implicit solvation model for density- 
functional study of nanocrystal surfaces and reaction pathways. J. Chem. Phys. 2014, 140, 084106.

(47) Nadeem, I. M.; Treacy, J. P. W.; Selcuk, S.; Torrelles, X.; Hussian, H.; Wilson, A.; Grinter, D. C.; Cabailh, G.; Bikondoa,
O.; Nicklin, C.; Selloni, A.; Zegenhagen, J.; Lindsay, R.; Thornton, G. Water Dissociates at the Aqueous Interface with Reduced Anatase TiO2 (101). J. Phys. Chem. Lett. 2018, 9 , 3131-3136.

Table 1. Predicted valence band top $\left(V_{b e}\right)$ of binary compounds using "Modified Vacuum Approach" for the given Fockexchange $(\alpha)$ percentage and their comparison with the available experimental data from available flat-band measurements at neutral $\mathrm{pH}$ and room temperature.

\begin{tabular}{|c|c|c|c|c|c|c|c|c|}
\hline \multirow{2}{*}{$\begin{array}{l}\text { Modified- } \\
\text { Vacuum } \\
\text { Approach }\end{array}$} & \multicolumn{2}{|c|}{$\begin{array}{c}\text { Experimental, } V_{b e} \\
(\mathrm{eV})\end{array}$} & \multicolumn{6}{|c|}{ Calculated, $V_{b e}(\mathrm{eV})$} \\
\hline & $\begin{array}{c}\text { Vs. } \\
\text { NHE }\end{array}$ & $\begin{array}{c}\text { Vs. } \\
\text { Vacuum }\end{array}$ & $\begin{array}{c}\alpha= \\
0.00 \%\end{array}$ & $\begin{array}{c}\text { Error vs. } \\
\text { Vacuum }(\mathrm{eV})\end{array}$ & $\begin{array}{c}\alpha= \\
25.00 \\
\%\end{array}$ & $\begin{array}{c}\text { Error vs. } \\
\text { Vacuum }(\mathrm{eV})\end{array}$ & $\begin{array}{c}\alpha= \\
63.50 \\
\%\end{array}$ & $\begin{array}{c}\text { Error vs. } \\
\text { Vacuum }(\mathrm{eV})\end{array}$ \\
\hline $\mathrm{SnO}_{2}$ & -3.89 & -8.39 & -5.44 & 2.95 & -6.46 & 1.93 & -8.03 & 0.36 \\
\hline $\mathrm{R}-\mathrm{TiO}_{2}$ & -3.11 & -7.61 & -4.91 & 2.70 & -5.82 & 1.79 & -7.24 & 0.37 \\
\hline $\mathrm{CdS}$ & -2.18 & -6.68 & -5.18 & 1.50 & -5.73 & 0.95 & -6.58 & 0.10 \\
\hline $\mathrm{A}-\mathrm{TiO}_{2}$ & -3.05 & -7.55 & -5.91 & 1.64 & -6.84 & 0.71 & -8.26 & -0.71 \\
\hline $\mathrm{ZnO}$ & -3.03 & -7.53 & -5.22 & 2.31 & -6.17 & 1.36 & -7.63 & -0.10 \\
\hline $\mathrm{SiC}$ & -1.72 & -6.22 & -5.79 & 0.43 & -6.38 & -0.16 & -7.18 & -0.96 \\
\hline $\mathrm{CdSe}$ & -1.66 & -6.16 & -5.15 & 1.01 & -5.63 & 0.53 & -6.38 & -0.22 \\
\hline InP & -1.33 & -5.83 & -4.81 & 1.02 & -5.16 & 0.67 & -5.69 & 0.14 \\
\hline $\mathrm{GaP}$ & -1.30 & -5.80 & -4.94 & 0.86 & -5.30 & 0.50 & -5.86 & -0.06 \\
\hline GaAs & -0.84 & -5.34 & -4.79 & 0.55 & -4.93 & 0.41 & -5.16 & 0.18 \\
\hline $\mathrm{ZnTe}$ & -1.02 & -5.52 & -4.81 & 0.71 & -5.25 & 0.27 & -5.93 & -0.41 \\
\hline MAE (SD) & - & - & \multicolumn{2}{|c|}{$1.43(0.88)$} & \multicolumn{2}{|c|}{$0.84(0.64)$} & \multicolumn{2}{|c|}{$0.33(0.43)$} \\
\hline
\end{tabular}

Table 2. Predicted valence band-edges $\left(V_{b e}\right)$ of binary compounds using "He-Slab Approach" for the given Fock-exchange $(\alpha)$ percentage and their comparison with the available experimental data from available flat-band measurements at neutral pH and room temperature.

\begin{tabular}{|c|c|c|c|c|c|c|c|c|}
\hline \multirow[t]{2}{*}{$\begin{array}{c}\text { He-Slab } \\
\text { Approach }\end{array}$} & \multicolumn{2}{|c|}{$\begin{array}{c}\text { Experimental, } V_{b e} \\
(\mathrm{eV})\end{array}$} & \multicolumn{6}{|c|}{ Calculated, $V_{b e}(\mathrm{eV})$} \\
\hline & $\begin{array}{c}\text { Vs. } \\
\text { NHE }\end{array}$ & $\begin{array}{c}\text { Vs. } \\
\text { Vacuum }\end{array}$ & $\begin{array}{c}\alpha= \\
0.00 \%\end{array}$ & $\begin{array}{c}\text { Error vs. } \\
\text { Vacuum }(\mathrm{eV})\end{array}$ & $\begin{array}{c}\alpha= \\
25.00 \\
\%\end{array}$ & $\begin{array}{c}\text { Error vs. } \\
\text { Vacuum }(\mathrm{eV})\end{array}$ & $\begin{array}{c}\alpha= \\
73.00 \\
\%\end{array}$ & $\begin{array}{c}\text { Error vs. } \\
\text { Vacuum }(e V)\end{array}$ \\
\hline $\mathrm{SnO}_{2}$ & -3.89 & -8.39 & -5.52 & 2.87 & -6.49 & 1.90 & -8.37 & 0.02 \\
\hline $\mathrm{R}-\mathrm{TiO}_{2}$ & -3.11 & -7.61 & -4.85 & 2.76 & -5.73 & 1.88 & -7.44 & 0.17 \\
\hline $\mathrm{CdS}$ & -2.18 & -6.68 & -5.15 & 1.53 & -5.68 & 1.00 & -6.70 & -0.02 \\
\hline $\mathrm{A}-\mathrm{TiO}_{2}$ & -3.05 & -7.55 & -5.32 & 2.23 & -6.19 & 1.36 & -7.88 & -0.33 \\
\hline $\mathrm{ZnO}$ & -3.03 & -7.53 & -5.24 & 2.29 & -5.79 & 1.56 & -7.37 & 0.16 \\
\hline $\mathrm{SiC}$ & -1.72 & -6.22 & -4.99 & 1.23 & -5.34 & 0.88 & -6.02 & 0.20 \\
\hline $\mathrm{CdSe}$ & -1.66 & -6.16 & -4.95 & 1.21 & -5.43 & 0.73 & -6.37 & -0.21 \\
\hline InP & -1.33 & -5.83 & -4.61 & 1.22 & -5.00 & 0.83 & -5.74 & 0.09 \\
\hline $\mathrm{GaP}$ & -1.30 & -5.80 & -4.75 & 1.05 & -5.20 & 0.60 & -6.07 & -0.27 \\
\hline GaAs & -0.84 & -5.34 & -4.74 & 0.60 & -4.97 & 0.37 & -5.42 & -0.08 \\
\hline $\mathrm{ZnTe}$ & -1.02 & -5.52 & -4.87 & 0.65 & -5.32 & 0.20 & -6.20 & -0.68 \\
\hline MAE (SD) & - & - & \multicolumn{2}{|c|}{$1.60(0.80)$} & \multicolumn{2}{|c|}{$1.03(0.58)$} & \multicolumn{2}{|c|}{$0.20(0.27)$} \\
\hline
\end{tabular}



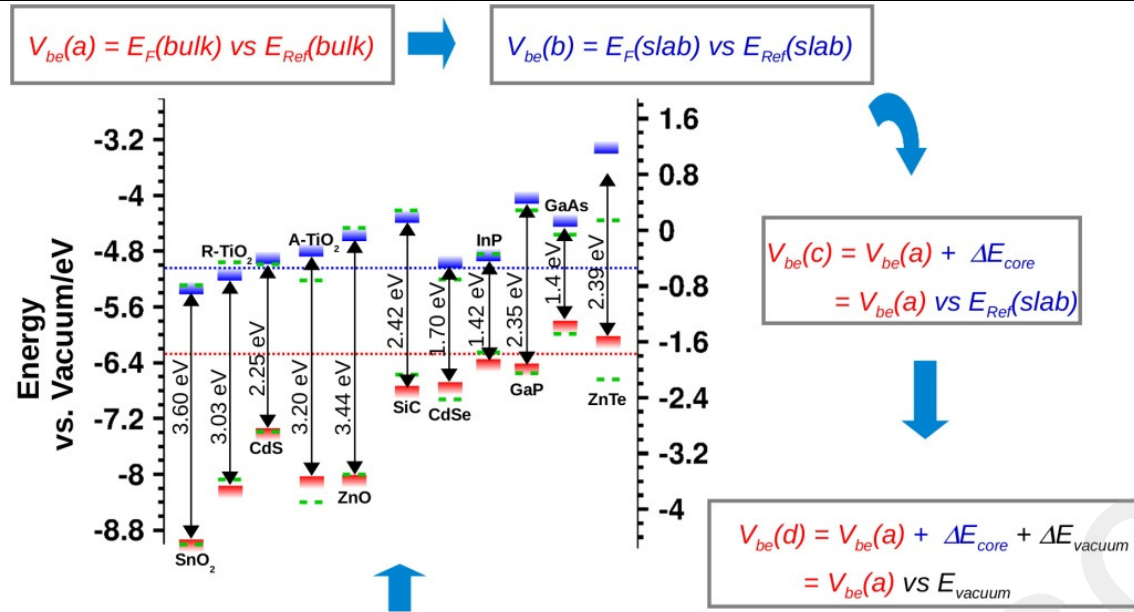

$V_{b e}(e)=V_{b e}(a)+\Delta E_{\text {core }}+\Delta E_{\text {vacuum }}+\Delta E_{H e}$ $=V_{\text {be }}(a) v s E_{\text {vacuum }}($ Corrected $)$ 


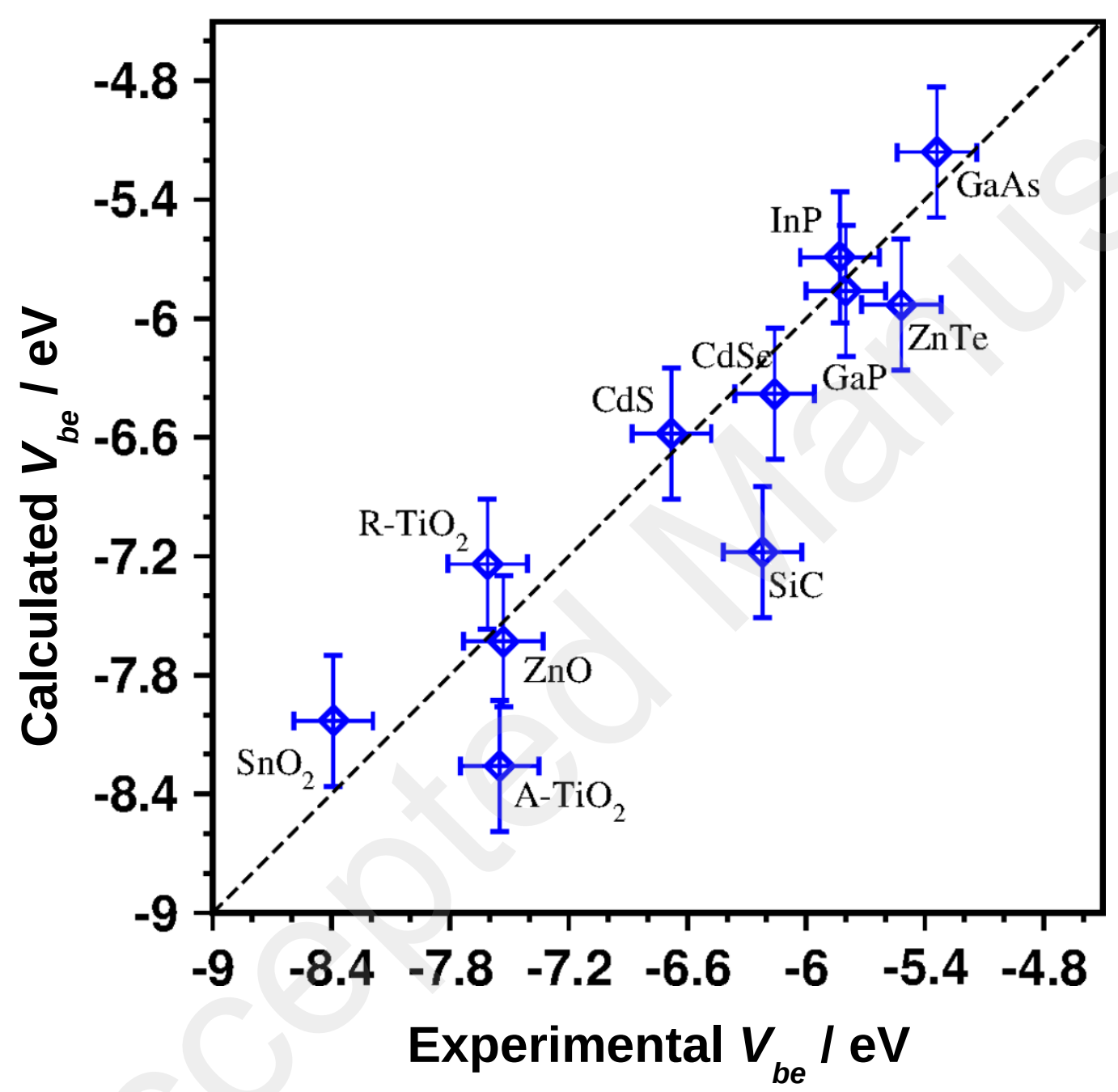




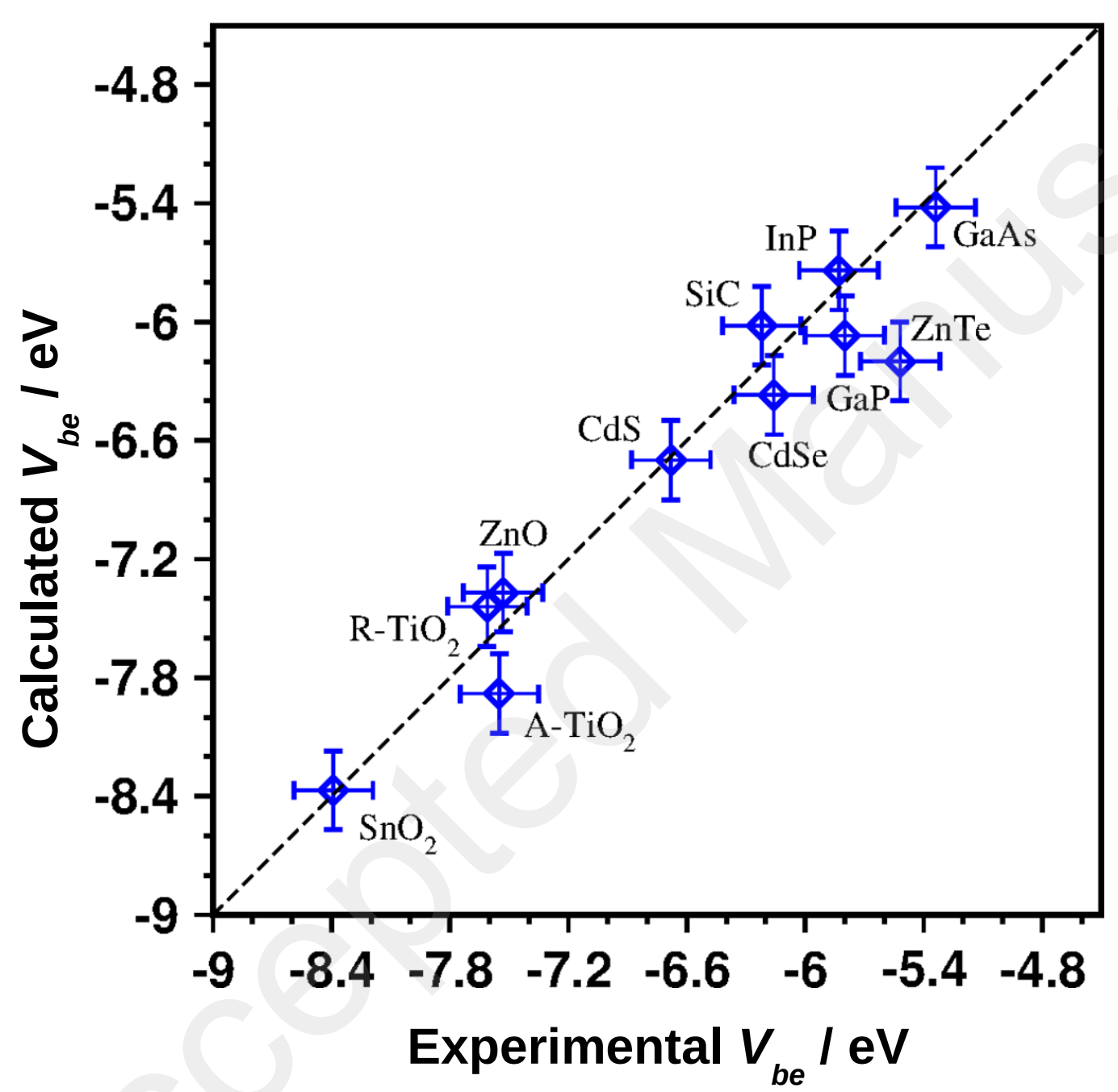




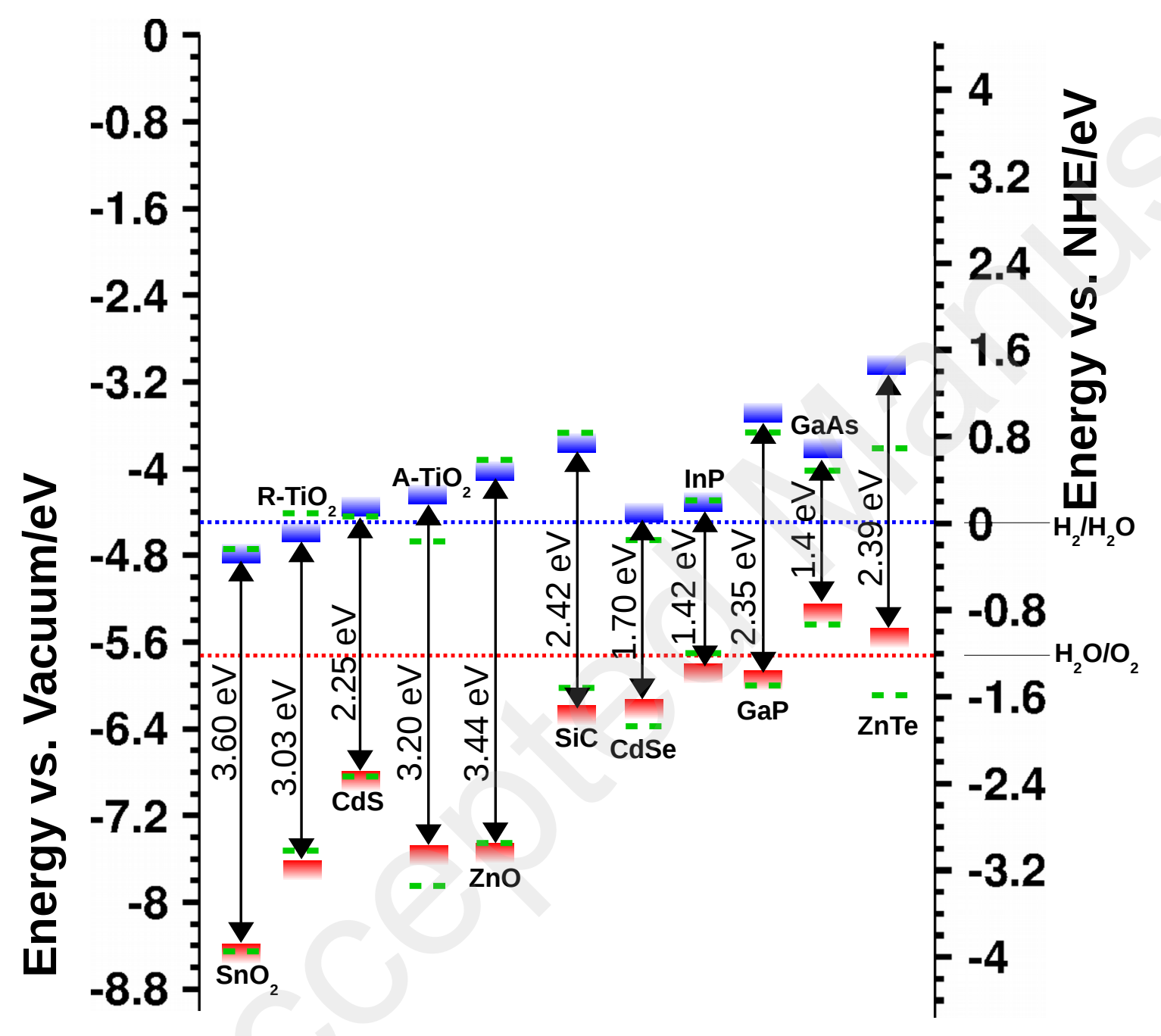

